\title{
Evaluating Breathing Discomfort in the ICU: What Is the Goal?
}

Patients on ventilators experience a variety of discomforts: symptoms (dyspnea, fatigue, pain), emotions (depression, anxiety, panic), and feeling a lack of control, to name a few. Patients may (or may not) express this discomfort outwardly with agitation, restlessness, grimacing, or increase in vital signs (breathing frequency, heart rate, blood pressure). Are these behaviors and measures signaling discomfort, and what is the source of this discomfort? For example, in the mechanically ventilated patient, are signs of discomfort, such as agitation displayed by the non-verbal patient, communicating distress related to the symptom of dyspnea or signs of irritation from an endotracheal tube? As caregivers, we all appreciate the importance of addressing the patient's source of discomfort while maintaining physiologic stability. But how does one evaluate discomfort? More importantly, how do we assess the cause of the discomfort to better identify interventions?

Dyspnea is one of several potential symptoms common in the mechanically ventilated patient in the ICU. However, not all patients receiving mechanical ventilation report dyspnea. Therefore, if we ask a patient receiving mechanical ventilation whether he or she is experiencing breathing discomfort (dyspnea), how do we know that the response about breathing is not a cry for help related to fear from being constrained by the ventilator? When patients are asynchronous with the ventilator, are they expressing panic and distress over the presence of an endotracheal tube, or does it reflect an underlying change in the patient's physiologic condition? Although getting to the bottom of patient distress is important, health-care providers have taken different approaches and sometimes have fallen short of this goal.

In a study of breathing difficulty, 96 subjects receiving mechanical ventilation who were able to respond to simple commands, were asked before enrollment, "Do you have trouble breathing?" ${ }^{2}$ Half of the subjects reported not experiencing dyspnea $(n=51,53 \%)$. Those with breathing difficulty were then asked to rate their intensity of respi-

\footnotetext{
The authors have disclosed no conflicts of interest.
}

Correspondence: Suzanne C Lareau RN MSc, College of Nursing, University of Colorado, Educ 2 North/Room 4327, 13120 East 19th Avenue, Aurora, CO 80045. E-mail: Suzanne.Lareau@ucdenver.edu.

DOI: $10.4187 /$ respcare. 05413 ratory discomfort on a $10-\mathrm{cm}$ visual analog scale anchored by "no respiratory discomfort" to "intolerable respiratory discomfort." This same scale was used for subjects to report their degree of anxiety and pain. Subjects who reported respiratory discomfort were also asked to choose whether the discomfort was associated with the terms air hunger, increased respiratory effort, or both.

\section{See the Original Study on Page 150}

In the study by Schmidt et al, ${ }^{1}$ the median visual analog scale rating of dyspnea was 5 (interquartile range 4-7), indicating moderate to intense discomfort. Of the 45 subjects expressing breathing discomfort, 28 selected a specific descriptor of their discomfort, with 15 selecting air hunger, 7 selecting respiratory effort, and 6 choosing both descriptors. The median rating of anxiety and pain, like dyspnea, was 5. Comparing the differences among those with $(n=45)$ and without $(n=51)$ dyspnea, anxiety was significantly more frequent $(P<.001)$ among those with dyspnea $(71 \%)$ than those without (24\%). Likewise, pain was more frequent ( $42 \%$ vs $18 \%$ ) among those with dyspnea than those without $(P=.01)$, whereas vital signs (heart rate, breathing frequency, and blood pressure) were not different between groups.

On further analysis of the results of Schmidt et al, ${ }^{1}$ dyspnea was strongly associated with anxiety (odds ratio 8.84, 95\% CI 3.26-24.0), continuous mandatory ventilation (odds ratio $4.77,95 \%$ CI $1.60-14.3$ ), and to a lesser degree heart rate (odds ratio 1.33 per 10 beats $/ \mathrm{min}, 95 \%$ CI 1.02-1.75). Those with dyspnea had a significantly longer stay (14 d) than those without dyspnea (8 d). Subjects reporting dyspnea also reported anxiety and pain more frequently than those without dyspnea, suggesting a relationship between these symptoms. In this study, ${ }^{1}$ subjectreported symptoms (not vital signs), were the strongest predictor of poor outcomes.

Campbell et $\mathrm{al}^{2,3}$ attempted to objectively assess dyspnea in subjects unable to communicate verbally, using a variety of cardiopulmonary parameters and observations of distress. These include observations and assessment of heart rate and breathing frequency, accessory muscle use, paradoxical breathing pattern, grunting (at end expiration), nasal flaring, restlessness, and a fearful facial display. ${ }^{2}$ Using these parameters, subjects admitted to an in-patient 


\section{EDITORIALS}

setting and referred for palliative care with a primary diagnosis of COPD, heart failure, and pneumonia were evaluated for their respiratory distress. ${ }^{3}$ Subjects were first asked "Are you short of breath?" followed by rating their distress on a visual analog scale (from 0 [no distress] to 10 [severe distress]). Of 136 subjects, over half $(53 \%, n=72)$ reported no shortness of breath. When presented with a scale to report their degree of dyspnea, many of these same subjects denying dyspnea reported moderate respiratory distress with a numerical rating of 5 ( $0-10$ scale). These findings suggest that patients denying dyspnea may still evaluate the intensity of dyspnea when presented with choices. Whether this was an attempt by the subject to please the study team or not is unclear; however, it shows the importance of establishing whether dyspnea exists before obtaining a numerical rating of distress or intensity.

Further support for concerns with objective ratings of respiratory distress was provided by a study of nurses and physicians rating a subject's breathlessness during a spontaneous breathing trial. ${ }^{4}$ This study demonstrated a systematic approach to identifying the presence of dyspnea, evaluation of its affective component, and determining the subject's feeling of resolution of the distress. Breathlessness was evaluated both by the patient and providers on a numeric rating scale of $0-10$. Subjects were asked the following questions: (1) "Do you have a sensation of breathlessness?" with options ranging from "No, not at all," to "Yes, worst imaginable"; (2) Do you feel secure during the spontaneous breathing trial?"; and (3) "Do you feel improvement of respiratory function?" anchored with "Yes, absolutely" and "No, not at all." Using the same metric, physicians and nurses were asked to independently rate the same questions for each subject. Of 100 subjects evaluated, breathlessness was rated as 5 (range 2-7), feeling insecure 3 (range 1-5), and improvement in respiratory function 4 (range 2-6). Physicians and nurses rated these 3 items as a 2 , which was significantly less $(P<.001)$ than subject ratings. Similar to other studies, many (38\%) reported experiencing no breathlessness to mild breathlessness $(<4$ on the numeric rating scale). In this trial, there were again a number of subjects not experiencing significant dyspnea, despite being exposed to the stress of the spontaneous breathing trial. Underscoring the affective dimension of dyspnea, breathlessness was negatively correlated with feeling secure $(\mathrm{r}=-0.50)$; thus, the more secure they felt, the less dyspnea they experienced.

In this issue, Binks et $\mathrm{al}^{5}$ sought to determine how well ICU clinicians were able to objectively assess a subject's breathing discomfort in 30 subjects receiving mechanical ventilation (during a sedation break). A research team member noted vital signs and ventilator settings and then asked the subject to rate his or her breathing discomfort on a modified Borg scale, anchored by 0 (no discomfort) to 10 (extremely uncomfortable). Within $15 \mathrm{~min}$ of this rating by the subject, a respiratory therapist, a registered nurse, and a physician (in 21 of 30 of cases) independently provided a numerical assessment of the subject's respiratory discomfort on a modified Borg scale. All subjects reported some breathing discomfort, with a median rating of 4 (range 4-7). The 3 types of health-care personnel failed to rate the same intensity as the subject's self-reported breathing discomfort, with physicians and respiratory therapists rating 1 point and nurses 2 points lower than the subject. These were all clinically important underestimations from the 1-point minimal clinically important difference on the Borg scale. ${ }^{6}$

Does this mean that the objective ratings of dyspnea by clinicians are of limited value? It should cause us to question these values for 2 reasons. First, dyspnea, as defined by the American Thoracic Society, is a subjective experience of breathing discomfort that consists of qualitatively distinct sensations that vary in intensity. The experience derives from interactions among multiple physiological, psychological, social, and environmental factors and may induce secondary physiological and behavioral responses. ${ }^{7}$ Symptoms have personal meaning to the patient that only the patient can understand. There are several dimensions to the sensation of dyspnea (intensity, affective component, and impact) that can be reported by the patient, any or all of which may be important at any given time. ${ }^{8}$ However, we limit our evaluation to the intensity of the patient's breathing distress without first establishing that breathing distress exists. In fact, we would obtain more information upon which to base interventions if we also evaluated the affective dimension, such as the degree of anxiety associated with dyspnea.

Second, do these objective assessments get clinicians closer to the goal of identifying the source of the patient's discomfort? Studies would indicate that we have no greater chance of estimating a patient's discomfort correctly, even when taking into account parameters such as breathing frequency. In the studies by Binks et $\mathrm{al}^{5}$ and Schmidt et $\mathrm{al},{ }^{1}$ breathing frequencies were within normal ranges, despite a moderate degree of breathing discomfort. Dyspnea, like pain, is a very subjective symptom. Why are we not just asking the patient?9,10 Only when the patient is unable to communicate in any available manner should the provider consider outward signs to guide determination of the source of discomfort. ${ }^{8}$ Many patients receiving mechanical ventilation are able to communicate, and surprisingly, many do not report dyspnea.

Care of the critically ill patient is a collaborative effort of the team of respiratory therapists, nurses, physical therapists, and physicians. Each brings a sometimes different view of the patient and evaluation of behaviors, all very important in decision making. In the patient unable to communicate, outward signs of distress are important red flags that need to be explored. For the respiratory thera- 


\section{EDITORIALS}

pist, restlessness may indicate a need to re-evaluate the ventilator settings; for the nurse, it may indicate a need to re-evaluate vital signs, patient position, and potential medications contributing to this behavior. However, these studies point to the danger of relying exclusively on objective information.

So what is the take-home message of these studies? First, given the fact that many patients receiving mechanical ventilation often can communicate, we need to work diligently at the bedside to determine the source of the patient's discomfort. This requires patience on the part of the provider to explore potential sources and the use of clinical judgment to rule out precipitating causes. Second, determining the intensity of symptoms by means other than obtaining information or ratings directly from the patient is potentially dangerous, leading to miscalculations and inappropriate interventions. If we assume that providers will underestimate patient ratings by $1-3$ points on a dyspnea scale, should we add this to our estimates? Any numerical rating should be put in the context of the source of discomfort. Third, once we determine the presence of dyspnea and its intensity, do we treat it? Does a rating of $8(0-10$ scale $)$ mean such severe dyspnea that we must intervene? To intervene therapeutically, one would have to then establish the quality of the dyspnea. Is the discomfort from respiratory effort (which would dictate an evaluation of ventilator settings, patency of airway), or does it reflect a fear of having breathing controlled by the ventilator (which would dictate a different approach to comforting the patient and potentially intervening with medication).

Assessing dyspnea, like pain, should be methodical. Assessment of dyspnea (discomfort/respiratory distress) should: (1) identify its presence, (2) determine the type of discomfort, (3) identify the level of discomfort dyspnea is creating, (4) identify the source of discomfort, (5) treat the discomfort, and (6) reassess whether the discomfort has been satisfactorily reduced. A good example of an instrument to evaluate the sensory and affective qualities of dyspnea is the multidimensional dyspnea profile. ${ }^{11}$ It consists of 4 questions that address the components of dyspnea by evaluating its intensity, unpleasantness, sensory qualities (eg, effort, hunger for air), and emotional response (eg, frustration, fear) to dyspnea. Systematic evaluations, founded on our knowledge of symptoms, will help us to reach our goal of addressing symptoms of patients requiring mechanical ventilation. Vital signs are only an alert that something is bothering the patient emotionally, physically, or physiologically. Dyspnea is a subjective symptom and thus requires more engagement with the patient to understand the experience and determine whether intervention is needed.

\section{Suzanne C Lareau RN MSc Mary Beth Makic PhD RN CNS CCNS}

College of Nursing

University of Colorado Aurora, Colorado

\section{REFERENCES}

1. Schmidt M, Demoule A, Polito A, Porchet R, Aboad J, Siami S, et al. Dyspnea in mechanically ventilated critically ill patients. Crit Care Med 2011;39(9):2059-2065.

2. Campbell ML, Templin T, Walch J. A respiratory distress observation scale for patients unable to self-report dyspnea. J Palliat Med 2010;13(3):285-290.

3. Campbell ML, Templin TN. Intensity cut-points for the respiratory distress observation scale. Palliat Med 2015;29(5):436-442.

4. Haugdahl HS, Storli SL, Meland B, Dybwik K, Romild U, Klepstad P. Underestimation of patient breathlessness by nurses and physicians during a spontaneous breathing trial. Am J Respir Crit Care Med 2015;192(12):1440-1448.

5. Binks A, Desjardins S, Riker R. ICU clinicians under-estimate breathing discomfort in ventilated patients. Respir Care 2017;62(2):150-155.

6. Ries AL. Minimally clinically important difference for the UCSD shortness of breath questionnaire, Borg scale, and visual analog scale. COPD 2005;2(1):105-110.

7. American Thoracic Society. Dyspnea: mechanisms, assessment, and management: a consensus statement. Am J Respir Crit Care Med 1999;159(1):321-340.

8. Parshall MB, Schwartzstein RM, Adams L, Banzett RB, Manning HL, Bourbeau J, et al. An official American Thoracic Society Statement: update on the mechanisms, assessment, and management of dyspnea. Am J Respir Crit Care Med 2012;185(4):435-452.

9. Schmidt M, Banzett RB, Raux M, Morélot-Panzini C, Dangers L, Similowski T, Demoule A. Unrecognized suffering in the ICU: addressing dyspnea in mechanically ventilated patients. Intensive Care Med 2014;40(1):1-10.

10. Banzett RB, Schwartzstein RM. Dyspnea: Don't just look, ask! Am J Respir Crit Care Med 2015;192(12):1404-1406.

11. Meek PM, Banzett R, Parshall MB, Gracely RH, Schwartzstein RM, Lansing R. Reliability and validity of the multidimensional dyspnea profile. Chest 2012 141(6):1546-1553. 